\title{
LA DIVERSIDAD DE CRITERIOS EN EL CONTROL CONTABLE GUBERNAMENTAL
}

\section{THE DIVERSITY OF DISCERNMENT ON THE CONTROL ACCOUNTING GOVERNMENTAL}

\section{Julio Flores Konja* \\ JuAn Miñano LeCAROS ${ }^{* *}$}

[Recepción: Abril de 2009 / Conformidad: Mayo de 2009]

\section{RESUMEN}

Los estados financieros, que periódicamente exponen las administraciones públicas en nuestro medio, reflejan la diversidad de criterios de control contable gubernamental, apartándose de la normativa presupuestaria, pues de acuerdo al artículo 77 de nuestra Constitución Política, el Estado administra un presupuesto, que es un flujo de efectivo, y su dinámica de ingresos y gastos es contabilizado y reflejado en los estados financieros.

Por ello, el alejamiento del principio contable gubernamental del "paralelismo y simultaneidad contable” es apreciado en una lectura a los estados financieros, que para el presente trabajo hemos tomado en consideración la información financiera contenida en la Cuenta General de la República del año 2006.

Las apreciaciones que exponemos permitirán la unificación de criterios en la elaboración y presentación de los estados financieros, tal como se prescribe en las diversas Normas Internacionales de Contabilidad para el Sector Público (NIC-SP).

Palabras clave: Criterios, control contable, presupuesto, NIC-SP, gestión contable.

\begin{abstract}
The financial states, a means of periodic public management in ours showing, reflect several discernment of accounting control governmental, to side on the regulatory budget, then to agree at $77^{\circ}$ article we ours Political Constitution, the public management at budget, the same is cash flow and dynamic ours of income and expense, is accountibily and reflecty to Financial States.

For that reason, away of doctrine accounting governmental of "parallelism and simultaneous ", is appraisal in ours readyng of Financial States, we hope present work to take into consideration, the information financial in to General Accounts of Republic from 2006.

The appreciations we exhibitions, to contribute at receive of critery unifications in to making and presentation from Financial States, that as the prescribe of International Accounting Standars of Governmental ( IAS-G ).
\end{abstract}

Keywords: Discernment, accounting control, budget, IAS-G, accounting governmental. 


\section{INTRODUCCIÓN}

Básicamente, la diversidad de criterios en el control gubernamental se refleja en la auditoría a los estados financieros. Tal es el caso del rubro de Caja y Banco, como parte integrante del Activo Corriente, en el Balance General. Sucede que de acuerdo a la normativa de ejecución presupuestaria ${ }^{1}$ y a la Directiva de Tesorería ${ }^{2}$, ambos obviamente de aplicación a las entidades gubernamentales, no es factible realizar mayor gasto de aquel presupuestado y que haya sido formalizado en las etapas de comprometido, devengado, girado y pagado.

Entonces, no puede darse la figura del sobregiro bancario y considerarse luego como parte integrante del pasivo corriente, tal como se realiza en la gestión privada. Sin embargo, en muchas oportunidades es aceptado y mostrado en el Balance General, tal es el caso que se refleja en la Cuenta General de la República del 2006, tanto a nivel de Gobierno Nacional y de Gobierno Local ${ }^{3}$.

Otro aspecto muy común, que evidencia la diversidad de criterios en la gestión contable, es la referente a las Cuentas por Cobrar, específicamente a aquellas deudas comprometidas en el año fiscal, pero que son pagadas en los primeros periodos del ejercicio fiscal siguiente. Siguiendo la lógica normativa presupuestaria, al 31 de diciembre se concluyen las gestiones presupuestarias de ingresos y gastos. Por consiguiente, aquello que es liquidado posteriormente a esa fecha debe ser mostrado como parte integrante del Activo no Corriente, es decir: Cuentas por Cobrar a Largo Plazo, sin embargo, muchas entidades lo exponen como parte integrante del Activo Corriente, bajo el argumento de haberse emitido el comprobante de pago en dicho año fiscal que acaba de concluir.

Respecto al Pasivo, en el sector del Pasivo Corriente, se expone el rubro "Obligaciones Tesoro Público", que como saldo contable no significa la obligación de pago de deuda alguna. Todo lo contrario, son saldos que contienen las deudas asumidas por la entidad pública con cargo a la fuente de financiamiento de Recursos Ordinarios, pero que los beneficiarios del mismo, no han hecho efectivo el cobro en el Banco de la Nación, pues, según el Estado Bancario del periodo, son cheques girados o abonos en Cuenta Corriente Interbancaria no cobrados. Pero dicha aplicación contenida en la dinámica contable de esa partida según el Nuevo Plan Contable Gubernamental, posterior al cobro del cheque o abono en Cuenta Corriente Interbancaria a favor del beneficiario, esta operación se reflejará en el Estado Bancario y recién la entidad podrá registrar como ingreso, por intermedio de la cuenta "Transferencias Corrientes", para ser mostrado en el Estado de Gestión.

Un rubro que también debe ser materia de una revisión es la "Reserva”, que se expone en el sector del Patrimonio, toda vez que la entidad no obtiene pérdida o ganancia al final del periodo. Lo que este último permitiría, en el supuesto de obtenerse superávit, es efectuar la reserva correspondiente.

Con relación al Estado de Gestión, hay dos aspectos centrales que exigen una reflexión para la unificación de criterios. El primero es lo relacionado al rubro "Transferencias Corrientes", que en el párrafo ante-

1 Directiva No 003-07-EF/7601, “Ejecución Presupuestaria en el Gobierno Nacional, Regional y Local”; Boletín de Normas Legales del Diario Oficial El Peruano, Lima 21 de enero del 2007.

2 Resolución Directoral No 02-07-EF/77.15, "Directiva de Tesorería”, "Boletín de Normas Legales del Diario Oficial El Peruano", Lima 27 de Enero de 2007.

3 Decreto Legislativo N 993, aprueban Cuenta General de la República del año 2006, Boletín de Normas Legales del Diario Oficial “El Peruano”, Lima, 21 de febrero del 2008, página 366978. 


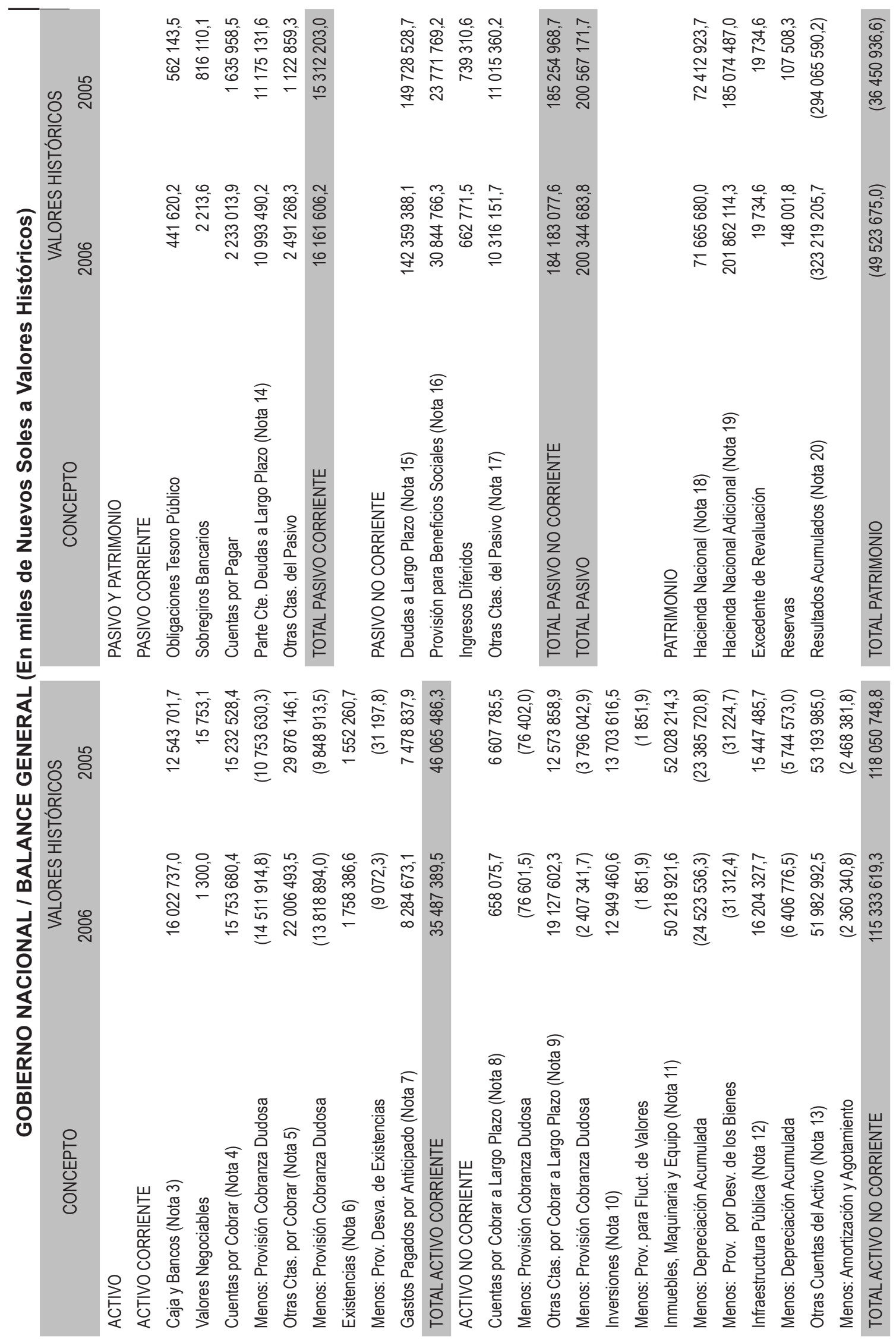




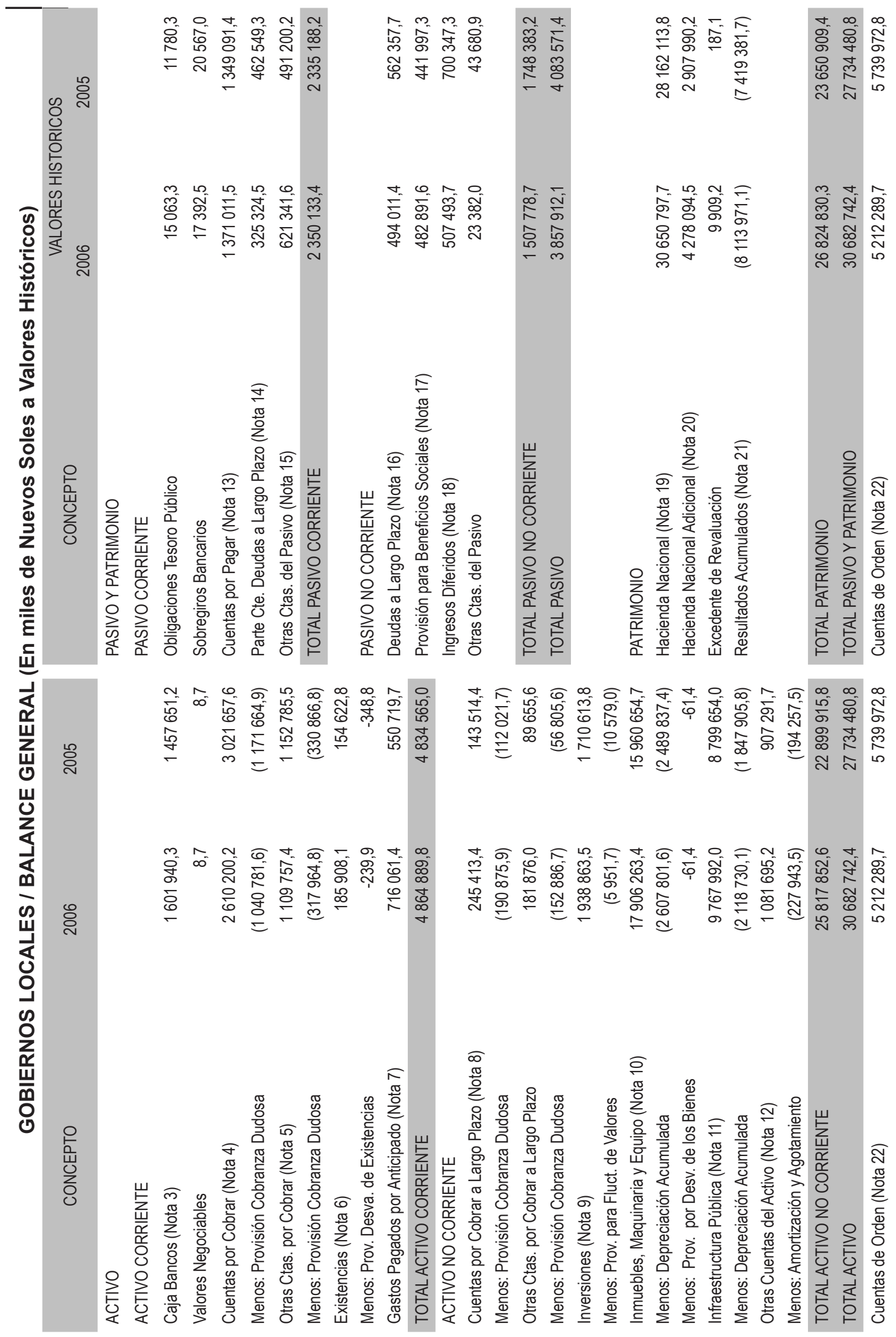

86/ QVIPURamavoc | Vol. 16(1) 2009 


\section{GOBIERNO NACIONAL}

ESTADO DE GESTIÓN

(En Miles de Nuevos Soles a Valores Históricos)

CUADRO $\mathrm{N}^{\circ} \mathrm{g}$

Por los años terminados al 31 de diciembre de:

\section{CONCEPTO} 2006 2005

\section{INGRESOS}

Ingresos Tributarios (Nota 22)

56997351,7

47779320,6

Menos: Liber. Inc. y Dev. Tributarios

(6 598 123,9)

(6593 295,9)

Ingresos No Tributarios y Ventas Netas

3756486,6

3290214,3

Transferencias Corrientes Recibidas (Nota 23)

62887,8

TOTAL INGRESOS

54218602,2

44476239,0

\section{COSTOS Y GASTOS}

Costo de Ventas

$(1337586,8)$

(1 099853,7$)$

Gastos de Ventas

$(8657,9)$

$(8219,9)$

Gastos Administrativos (Nota 24)

(6 083201,5$)$

(5 434458,4$)$

Gastos de Personal (Nota 25)

(6 994026,4$)$

(6 433 902,7)

Provisiones del Ejercicio (Nota 26)

(39 145 348,3)

(34 395 790,5)

TOTAL COSTOS Y GASTOS

(53568 820,9)

(47 372 225,2)

RESULTADO OPERACIONAL

649781,3

(2 895 986,2)

\section{OTROS INGRESOS Y GASTOS}

Ingresos Financieros (Nota 27)

11064726,6

9727660,4

Ingresos Diversos de Gestión

1102338,0

2686818,0

Gastos Div. de Gestión y Subv. Otorgadas (Nota 28)

(5 941 163,3)

(7 947788,1$)$

Gastos Financieros (Nota 29)

(10 278525,3$)$

(9 639464,3$)$

Transferencias Ctes. Otorgadas (Nota 30)

$(9513,1)$

Ingresos Extraordinarios (Nota 31)

2726382,2

1221169,7

Gastos Extraordinarios (Nota 32)

(2 237386,0$)$

(1559 034,5)

Ingresos de Ejercicios Anteriores (Nota 33)

26054251,8

11932932,7

Gastos de Ejercicios Anteriores (Nota 34)

(19 883743,0$)$

(4 621249,8$)$

Otros Ingresos

64094,2

49004,9

Otros Ingresos

$(130031,4)$

$(219242,8)$

REIE (898)

TOTAL OTROS INGRESOS Y GASTOS

2531430,7

1630806,2

RESULT. DEL EJERCICIO SUPERAVIT (DÉFICIT)

3181212,0

$(1265180,0)$

Vol. 16(1) 2009 | QUIPURAMAVOC / 87 


\section{GOBIERNOS LOCALES \\ ESTADO DE GESTIÓN}

(En Miles de Nuevos Soles a Valores Históricos)

CUADRO N ${ }^{\circ} 34$

Por los años terminados al 31 de diciembre de:

\section{CONCEPTO} 2006 2005

\begin{tabular}{|c|c|c|}
\hline CONCEPTO & 2006 & 2005 \\
\hline \multicolumn{3}{|l|}{ INGRESOS } \\
\hline Ingresos Tributarios (Nota 23) & 1910503,4 & 1879481,4 \\
\hline Menos: Liber. Inc. y Dev. Tributarios & $(30135,3)$ & $(20181,8)$ \\
\hline Ingresos No Tributarios (Nota 24) & 803183,4 & 908854,0 \\
\hline Transferencias Corrientes Recibidas (Nota 25) & 1903851,1 & 1566805,2 \\
\hline TOTAL INGRESOS & 4587402,6 & 4334958,8 \\
\hline \multicolumn{3}{|l|}{ COSTOS Y GASTOS } \\
\hline Costo de Ventas & $(51$ 175, $)$ & $(49362,3)$ \\
\hline Gastos de Ventas & $(44932,2)$ & $(31383,9)$ \\
\hline Gastos Administrativos (Nota 26) & $(1863939,9)$ & $(1631179,1)$ \\
\hline Gastos de Personal (Nota 27) & $(997907,2)$ & $(965941,5)$ \\
\hline Provisiones del Ejercicio (Nota 28) & $(1245908,1)$ & $(1241397,3)$ \\
\hline TOTAL COSTOS Y GASTOS & (4 203862,5$)$ & (3 919264,1$)$ \\
\hline RESULTADO OPERACIONAL & 383540,1 & 415694,7 \\
\hline \multicolumn{3}{|l|}{ OTROS INGRESOS Y GASTOS } \\
\hline Ingresos Financieros & 42092,3 & 65498,6 \\
\hline Ingresos Diversos de Gestión & 41072,4 & 30280,5 \\
\hline Gastos Div. de Gestión y Subv. Otorgadas (Nota 29) & $(595931,2)$ & $(632394,0)$ \\
\hline Gastos Financieros & $(90022,7)$ & $(111577,9)$ \\
\hline Transferencias Ctes. Otorgadas (Nota 30) & $(138132,2)$ & $(121356,8)$ \\
\hline Ingresos Extraordinarios & 67170,7 & 109201,4 \\
\hline Gastos Extraordinarios & $(50916,2)$ & $(81999,1)$ \\
\hline Ingresos de Ejercicios Anteriores (Nota 31) & 643129,9 & 639968,7 \\
\hline Gastos de Ejercicios Anteriores (Nota 32) & $(694852,5)$ & $(536993,6)$ \\
\hline Otros Ingresos & 10724,1 & 2208,0 \\
\hline Otros Egresos & $(83335,7)$ & $(45504,9)$ \\
\hline TOTAL OTROS INGRESOS Y GASTOS & $(849001,1)$ & $(682669,1)$ \\
\hline RESULT. DEL EJERCICIO SUPERAVIT (DÉFICIT) & $(465461,0)$ & $(266974,4)$ \\
\hline
\end{tabular}

88/ QVIPVIRAMAYoc | Vol. 16(1) 2009 


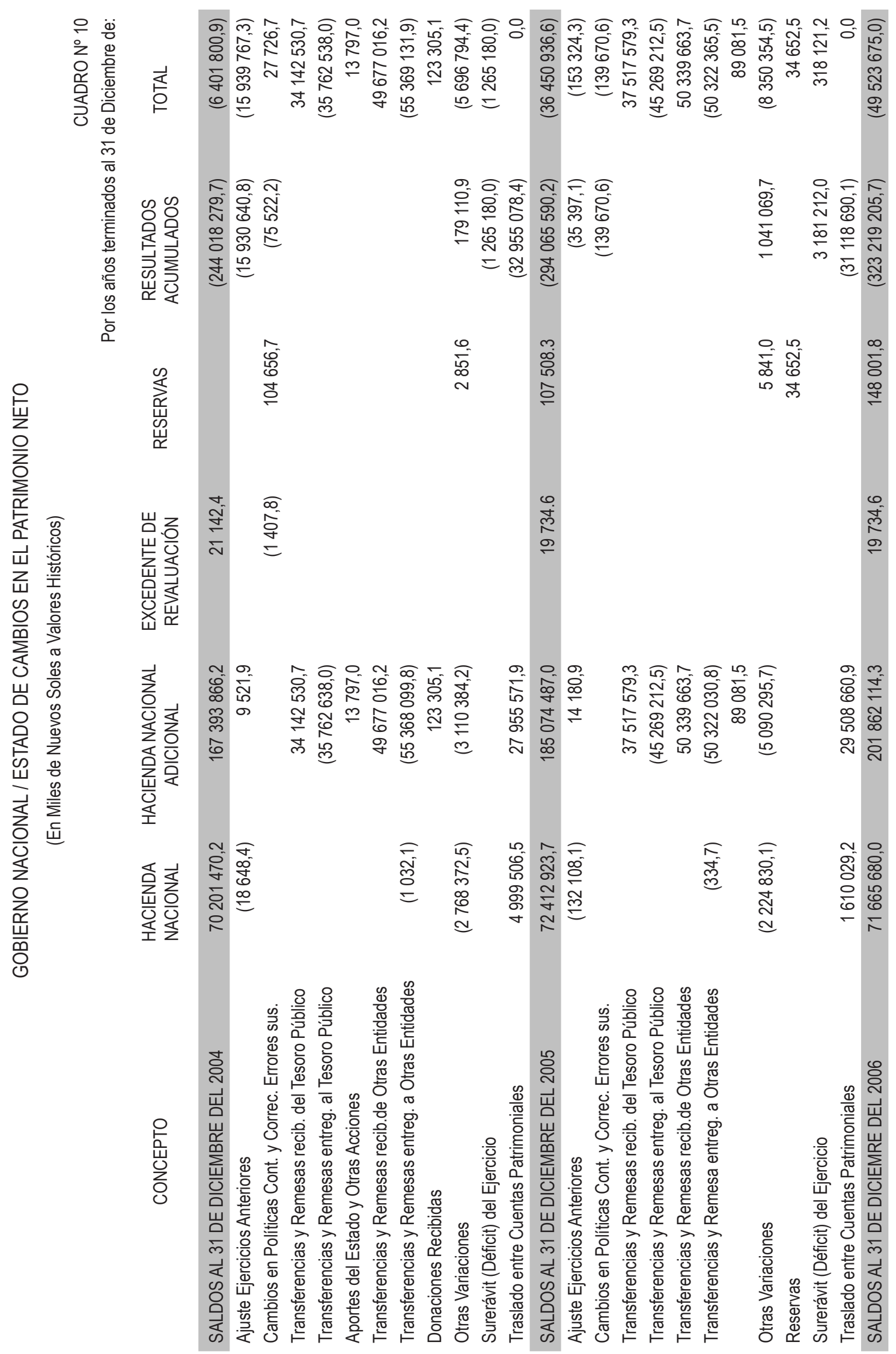




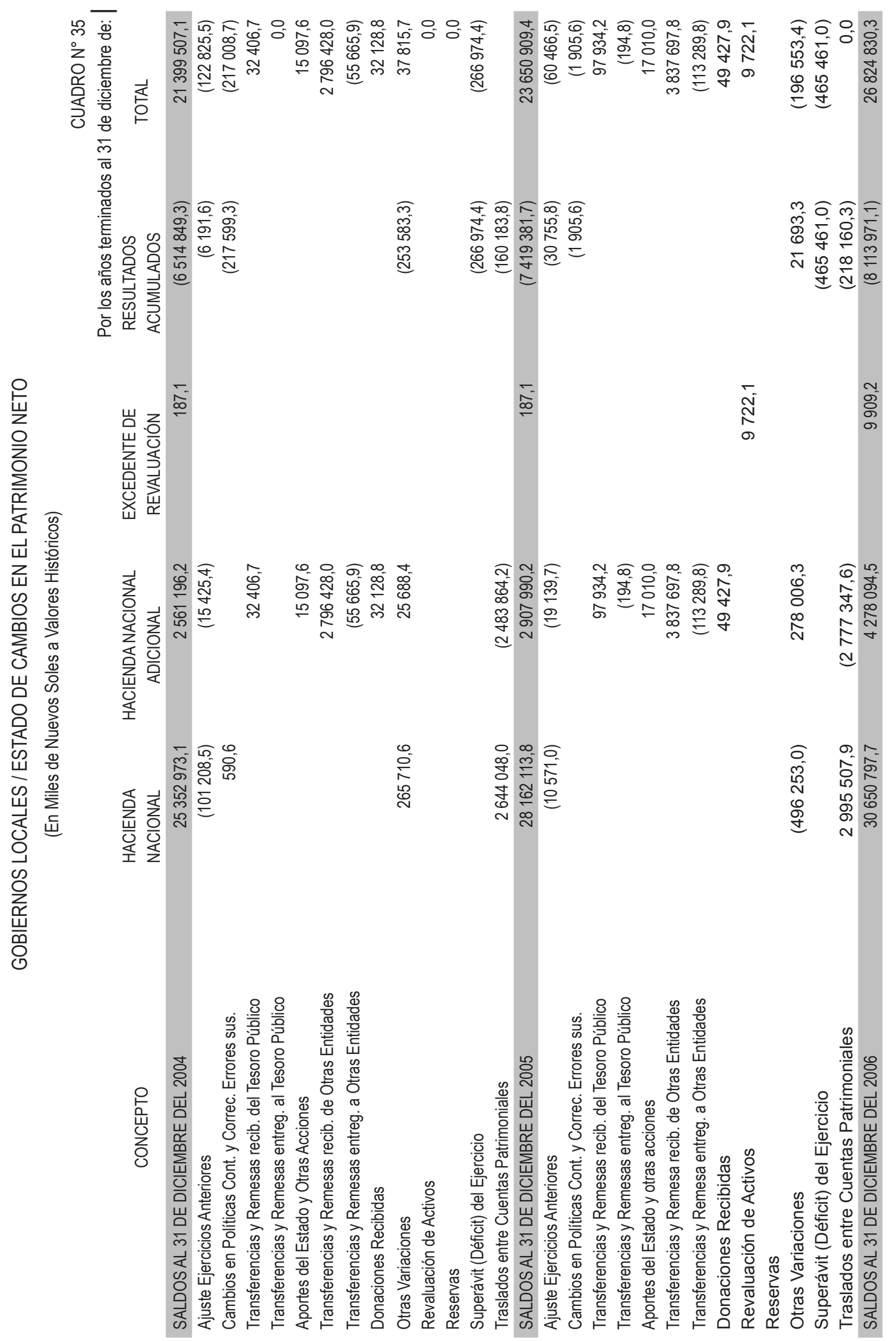




\section{GOBIERNO NACIONAL / ESTADO DE FLUJOS DE EFECTIVO}

(En Miles de Nuevos Soles a Valores Históricos)

Cuadro $\mathrm{N}^{\circ} 11$

Por los años terminados al 31 de diciembre del:

\begin{tabular}{|c|c|c|}
\hline CONCEPTO & 2006 & 2005 \\
\hline \multicolumn{3}{|l|}{ A. ACTIVIDADES DE OPERACIÓN } \\
\hline Cobranza de Impuestos, Tasas y Contribuciones & 51737817,9 & 41199157,3 \\
\hline Cobranza de Venta de Bienes y Servicios y Rentas de la Propiedad & 3976660,1 & 3628021,6 \\
\hline Donaciones Corrientes en Efectivo & 80687,0 & 73423,4 \\
\hline Transferencias Corrientes Recibidas & 62887,8 & 9840,0 \\
\hline Otros & 4308913,0 & 4056125,8 \\
\hline \multicolumn{3}{|l|}{ REIE (Entrada de Fondos) } \\
\hline \multicolumn{3}{|l|}{ MENOS } \\
\hline Pago a Proveedores de Bienes y Servicios & $(5657578,9)$ & (5 304 485,5) \\
\hline Pago de Remuneraciones y Obligaciones Sociales & $(7472864,0)$ & $(6925455,0)$ \\
\hline Pago de Pensiones y Otros Beneficios & $(7296180,1)$ & (6 119937,5$)$ \\
\hline Transferencias Corrientes Entregadas & $(9513,1)$ & $(2419,4)$ \\
\hline Pagos de Tributos & $(57419,8)$ & $(65543,3)$ \\
\hline Otros & $(13657$ 138,8) & $(12711648,1)$ \\
\hline \multicolumn{3}{|l|}{ REIE (Salida de Fondos) } \\
\hline $\begin{array}{l}\text { AUMENTO (DISMINUCIÓN) DEL EFECTIVO Y EQUIVALENTE DE } \\
\text { EFECTIVO PROVENIENTE DE LAACTIVIDAD DE OPERACIÓN }\end{array}$ & 26016271,1 & 17841918,1 \\
\hline \multicolumn{3}{|l|}{ B. ACTIVIDADES DE INVERSION } \\
\hline Cobranza de Venta de Valores e Inversiones & 293,0 & 28941,1 \\
\hline Cobranza de Venta de Inmuebles, Maquinaria y Equipo & 7595,8 & 11376,3 \\
\hline Cobranza de Venta de Otras Cuentas del Activo & 141,7 & 872,5 \\
\hline Otros & 280653,9 & 201553,9 \\
\hline \multicolumn{3}{|l|}{ MENOS } \\
\hline Pago por Compra de Valores e Inversiones & $(349,2)$ & $(446,9)$ \\
\hline Pago por Compra de Inmuebles, Maquinaria y Equipo & $(656365,5)$ & $(728033,7)$ \\
\hline Pago en Construcciones en Curso & $(871973,8)$ & $(1236177,9)$ \\
\hline Pago por Compras de Otras Cuentas del Activo & $(773791,8)$ & $(968302,0)$ \\
\hline Otros & $(1883335,9)$ & $(1962828,8)$ \\
\hline $\begin{array}{l}\text { AUMENTO (DISMINUCIÓN) DEL EFECTIVO Y EQUIVALENTE DE } \\
\text { EFECTIVO PROVENIENTE DE LAACTIVIDAD DE INVERSION }\end{array}$ & (3 897 131,8) & $(4653045,5)$ \\
\hline \multicolumn{3}{|l|}{ C. ACTIVIDADES DE FINANCIAMIENTO } \\
\hline Cobranza de Emisión de Acciones & & 13797,0 \\
\hline Cobranza por Recursos Obtenidos de Emisión & 855,7 & 1438,2 \\
\hline Donaciones de Capital en Efectivo & 53880,4 & 75978,1 \\
\hline Transferencias de Capital Recibidas & 79483906,8 & 77720857,0 \\
\hline Préstamos Internos y/o Externos & 587306,8 & 750149,8 \\
\hline Otros & 946307,9 & 531381,6 \\
\hline \multicolumn{3}{|l|}{ MENOS } \\
\hline Transferencias de Capital Entregadas & $(87993978,2)$ & $(82087928,8)$ \\
\hline Amortización de Préstamos e Intereses & $(9964587,6)$ & $(9733779,1)$ \\
\hline Otros & $(1495462,0)$ & $(549141,0)$ \\
\hline $\begin{array}{l}\text { AUMENTO (DISMINUCIÓN) DEL EFECTIVO Y EQUIVALENTE DE } \\
\text { EFECTIVO PROVENIENTE DE LAACTIVIDAD DE FINANCIAMIENTO }\end{array}$ & $(18381770,2)$ & $(13277247,2)$ \\
\hline D. AUMENTO (DISMINUCIÓN) DEL EFECTIVO Y EQUIVALENTE DEL EFECTIVO & 3737369,1 & $(88374,6)$ \\
\hline E. SALDO EFECTIVO Y EQUIVALENTE DE EFECTIVO AL INICIO DEL EJERCICIO & 12285367,9 & 12632076,3 \\
\hline F. SALDO EFECTIVO Y EQUIVALENTE DEL EFECTIVO AL FINALIZAR EL EJERCICIO & 16022737,0 & 12543701,7 \\
\hline
\end{tabular}


GOBIERNOS LOCALES / ESTADO DE FLUJOS DE EFECTIVO

(En Miles de Nuevos Soles a Valores Históricos)

Cuadro $\mathrm{N}^{\circ} 36$

Por los años terminados al 31 de diciembre del:

\begin{tabular}{|c|c|c|}
\hline CONCEPTO & 2006 & 2005 \\
\hline \multicolumn{3}{|l|}{ A. ACTIVIDADES DE OPERACIÓN } \\
\hline Cobranza de Impuestos, Tasas y Contribuciones & 1698212,00 & 1692524,0 \\
\hline Cobranza de Venta de Bienes y Servicios y Rentas de la Propiedad & 744566,9 & 688631,0 \\
\hline Donaciones Corrientes en Efectivo & 24300,1 & 9839,7 \\
\hline Transferencias Corrientes Recibidas & 1903798,8 & 1566800,3 \\
\hline Otros & 111155,4 & 132754,6 \\
\hline \multicolumn{3}{|l|}{ MENOS } \\
\hline Pago a Proveedores de Bienes y Servicios & $(2536780,0)$ & (2 212 182,4) \\
\hline Pago de Remuneraciones y Obligaciones Sociales & $(1073459,2)$ & $(987105,3)$ \\
\hline Pago de Pensiones y Otros Beneficios & $(222770,6)$ & $(224935,8)$ \\
\hline Transferencias Corrientes Entregadas & $(138132,2)$ & $(121336,2)$ \\
\hline Pagos de Tributos & $(3423,2)$ & $(3448,4)$ \\
\hline Otros & $(422123,0)$ & $(348724,3)$ \\
\hline AUMENTO (DISMINUCIÓN) DEL EFECTIVO Y EQUIVALENTE DE & \multirow{2}{*}{85345,0} & \multirow{2}{*}{192817,2} \\
\hline EFECTIVO PROVENIENTE DE LAACTIVIDAD DE OPERACIÓN & & \\
\hline \multicolumn{3}{|l|}{ B. ACTIVIDADES DE INVERSIÓN } \\
\hline Cobranza de Venta de Inmuebles, Maquinaria y Equipo & 7768,2 & 8410,1 \\
\hline Cobranza de Venta de Otras Cuentas del Activo & 414,6 & 3071,0 \\
\hline Otros & 13691,3 & 25796,0 \\
\hline \multicolumn{3}{|l|}{ MENOS } \\
\hline Pago por Compra de Inmuebles, Maquinaria y Equipo & $(246$ 189,8) & $(176729,2)$ \\
\hline Pago en Construcciones en Curso & (2 954284,7$)$ & $(1747988,5)$ \\
\hline Pago por Compras de Otras Cuentas del Activo & $(181819,2)$ & $(148695,0)$ \\
\hline Otros & $(210279,8)$ & $(79631,3)$ \\
\hline AUMENTO (DISMINUCIÓN) DEL EFECTIVO Y EQUIVALENTE DE & \multirow{2}{*}{ (3 570699,4$)$} & \multirow{2}{*}{$(2115766,9)$} \\
\hline EFECTIVO PROVENIENTE DE LAACTIVIDAD DE INVERSION & & \\
\hline \multicolumn{3}{|l|}{ C. ACTIVIDADES DE FINANCIAMIENTO } \\
\hline Cobranza de Emisión de Acciones & 17510,2 & 8100,9 \\
\hline Cobranza por Recursos Obtenidos por emisión & 3871,3 & 4104,6 \\
\hline Donaciones de Capital en Efectivo & 42562,4 & 32077,3 \\
\hline Transferencias de Capital Recibidas & 3899537,1 & 2788142,0 \\
\hline Préstamos Internos y/o Externos & 42880,3 & 173441,8 \\
\hline Otros & 83986,5 & 33141,3 \\
\hline \multicolumn{3}{|l|}{ MENOS } \\
\hline Transferencias de Capital Entregadas & $(21473,7)$ & $(37708,2)$ \\
\hline Amortización de Préstamos e Intereses & $(301756,3)$ & $(418586,4)$ \\
\hline Otros & $(114672,0)$ & $(37342,1)$ \\
\hline AUMENTO (DISMINUCIÓN) DEL EFECTIVO Y EQUIVALENTE DE & 36524458 & 25152712 \\
\hline EFECTIVO PROVENIENTE DE LAACTIVIDAD DE FINANCIAMIENTO & $305 \angle 440,0$ & 2045311,2 \\
\hline D. AUMENTO (DISMINUCIÓN) DEL EFECTIVO Y EQUIVALENTE DEL EFECTIVO & 167091,4 & 622421,5 \\
\hline E. SALDO EFECTIVO Y EQUIVALENTE DE EFECTIVO AL INICIO DEL EJERCICIO & 1434848,9 & 835229,7 \\
\hline F. SALDO EFECTIVO Y EQUIVALENTE DEL EFECTIVO AL FINALIZAR EL EJERCICIO & 1601940,3 & 1457651,2 \\
\hline
\end{tabular}

92/ QVIPURAMAYOC | Vol. 16(1) 2009 
rior hemos expuesto ampliamente y el otro se relaciona con el rubro de "Provisiones del Ejercicio", pues son prácticas contables comunes exigidas por la dinámica de cuentas del nuevo plan contable gubernamental.

Las provisiones contables del periodo son cinco: Provisión de Cuentas de Cobranza Dudosa, Provisión de Desvalorización de Existencias, Provisión de Depreciación, Agotamiento de Inmuebles, Maquinaria y Equipo, Provisión de Amortización de Intangibles y Provisión de Compensación por Tiempo de Servicio.

Obviamente, estas prácticas contables no están presupuestadas y, por otro lado, no significa una salida de dinero de carácter inmediato. Sin embargo, su exposición en el Estado de Gestión incide en el resultado del periodo. Consecuentemente, la mayoría de las entidades públicas, expone como resultado del periodo déficit y asimismo en el Balance General, Sector del Patrimonio, se aprecia déficit en el rubro "Resultados Acumulados", en tal sentido, no significa que la entidad "sea gastadora" o refleje "pérdidas". Para una mejor ilustración, podemos apreciar el Estado de Gestión del Gobierno Nacional, así como también el correspondiente a los Gobiernos Locales, expuesta en la Cuenta General de la República del 2006, aprobado por el Decreto Legislativo $\mathrm{N}^{\circ} 993 .{ }^{4}$

Respecto al Estado de Cambios en el Patrimonio Neto, se aprecian dos aspectos trascendentales, uno relacionado al rubro "Reservas", que ya lo hemos tratado en el Balance General, y el otro, relacionado al rubro "Resultados Acumulados", que también lo hemos tratado anteriormente.
Sin embargo, en el Estado de Cambios en el Patrimonio Neto del Gobierno Nacional, que forma parte integrante de la Cuenta General de la República del 2006, se aprecia que el rubro "Reservas", expone saldo. Y por otro lado, el rubro "Resultados Acumulados", expone el déficit de S/.323’219,205.70, el cual como hemos expuesto anteriormente, no significa "Pérdida". En igual sentido para las entidades de los Gobiernos Locales, para el mismo periodo: Reservas S/.0.00, Resultados Acumulados S/. (8'113,971.10). ${ }^{5}$

El Estado de Flujos de Efectivo es sencillamente un Libro Auxiliar de Caja y Bancos. Los ingresos y gastos están clasificados por actividades de operación (ingresos y gastos corrientes), por actividades de inversión (ingresos y gastos por inmuebles, maquinarias y equipo y otras cuentas del activo), y por actividades de financiamiento (ingresos y gastos por préstamos e intereses).

Sin embargo, cabe precisar que en el rubro de ingresos, la dinámica contable de la partida: "Transferencia Corriente", "Obligaciones Tesoro Público" y "Sobregiro Bancario", obviamente incide en el resultado final del Estado de Flujos de efectivo, que debe ser igual a lo que se expone en el rubro Caja y Banco del Balance General.

Una explícita apreciación de lo manifestado, lo podemos evidenciar en el Estado de Flujos de Efectivo a nivel de Gobierno Nacional y de Gobierno Local, que se expone en la Cuenta General de la República correspondiente al año 2006, el mismo que cuando se compatibilizan los saldos entre el rubro Caja y Bancos, es igual al saldo de efectivo y equivalente de efectivo al finalizar el ejercicio

4 Decreto Legislativo N 993, aprueban Cuenta General de la República correspondiente al año 2006, Boletín de Normas Legales del Diario Oficial “El Peruano", Lima, 21 de febrero del 2008, página 3669782 Resolución Directoral No 0207-EF/77.15, "Directiva de Tesorería", "Boletín de Normas Legales del Diario Oficial El Peruano”, Lima 27 de Enero de 2007.

5 Decreto Legislativo N 993, aprueban Cuenta General de la República del año 2006, Boletín de Normas Legales del Diario Oficial “El Peruano", Lima, 21 de febrero del 2008, página 366978. 
que muestra los Estados de Flujos de Efectivo de las entidades públicas respectivamente indicados. ${ }^{6}$

\section{CONCLUSIONES}

La existencia de diversos criterios en el control contable gubernamental se refleja por:

Inexistencia de un Centro Superior de Gestión Pública, donde los funcionarios y servidores públicos, serían capacitados periódicamente en temas de presupuesto, tesorería y contabilidad, entre otros.

La ausencia de difusión de las Normas Internacionales de Contabilidad para el Sector Público ( NIC-SP )

La ausencia de difusión de normas y regulaciones de presupuesto, tesorería y contabilidad.

La errónea percepción de que los criterios y prácticas contables del sector privado tienen la misma equivalencia en el sector público. Tal es el caso de las llamadas "provisiones", que en la gestión pública deviene de la cobranza dudosa, la desvalorización de existencias, la depreciación de bienes del activo fijo, la amortización de intangibles y la compensación por tiempo de servicios.
Sin embargo, en la gestión privada, la práctica contable de "provisión", tiene una connotación de carácter permanente, por ejemplo, si se tiene un ingreso o gasto, se debe realizar primeramente la "provisión", lo que obviamente tiene un impacto en los estados financieros, específicamente, en aquel que muestra el resultado del periodo.

\section{REFERENCIAS BIBLIOGRÁFICAS}

1. Constitución Política del Perú, Separata Especial Diario El Peruano, Lima, diciembre de 1993.

2. Directiva No. 003-2007-EF/76.01, "Ejecución Presupuestaria en el Gobierno Nacional, Regional y Local.

3. Resolución Directoral $\mathrm{N}^{\circ}$. 02-2007EF/77.15, "Directiva de Tesorería”.

4. Decreto Legislativo Nº.993, Cuenta General de la República 2006.

5. Normas Internacionales de Contabilidad-Sector Público, volumen del I al VIII, Dirección Nacional de Contabilidad Pública.

6 Decreto Legislativo N 993, aprueban Cuenta General de la República del año 2006, Boletín de Normas Legales del Diario Oficial “El Peruano", Lima, 21 de febrero del 2008, página 366978. 